\title{
Oneiric stress and safety and security at work: the discovery of a new universal symbol
}

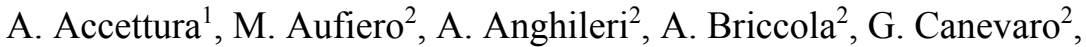 \\ N. Catelli ${ }^{1}$, M. Cavalleri ${ }^{2}$, R. Cusani ${ }^{1}$, E. De Lucia ${ }^{1}$, F. Borghini ${ }^{1}$, \\ F. Garzia ${ }^{1,3}$, M. Lombardi ${ }^{1}$, P. Luraschi ${ }^{2}$, M. Paddeu ${ }^{2} \&$ G. Rossi ${ }^{1}$ \\ ${ }^{1}$ Safety and Security Engineering, SAPIENZA, University of Rome, Italy \\ ${ }^{2}$ Sicuritalia S.p.A, Italy \\ ${ }^{3}$ Wessex Institute of Technology, UK
}

\begin{abstract}
Cox and Griffiths define as psychosocial risks at work "those aspects of the planning, organization and management of work, which, along with their environmental and social contexts, may affect mental and physical health of the employees, directly or indirectly producing stress". Therefore, a more effective approach to occupational safety and security should include integrated risk management through the identification of any work stress related problem. The purpose of this paper is to analyze the possible correlation of risk at work with the modification of sleep, and inside it, the specific function of dream activity. Keywords: occupational safety and security, work related stress, dream activity.
\end{abstract}

\section{Introduction}

The four main protagonists of our research are:

a) risk at work: over the years '70-'80 the public opinion started to pay attention to "health prevention and protection", then has moved its focus to the so-called "health promotion". In this new context, the health of workers is intended not only as the absence of disease or injury, but rather as the existence of a physical and psychological wellbeing state. In the mid $80 \mathrm{~s}$, the theme of risk at work gets the interest of the psychological sciences, and finally in recent years the organizational sciences too have focused its economic variables and business aspects. Today, organizational well-being commonly means the ability of an 
organization to promote and maintain workers' physical, psychological and social well-being for all its levels and roles. On 1986 the International Work Organization defined the concept of psychosocial risks later perfected by Cox and coworkers as "those aspects of the planning, organization and management of work, which, along with their environmental and social contexts, may affect mental and physical health of the employees, directly or indirectly producing stress" [1,2]. Therefore, psychosocial risks can directly and indirectly affect both physical and mental health through the experience of stress and its psychopathological work-related consequences: burnout [3] and mobbing [4].

b) stress: the term stress is known for centuries (starting from 1300-1400) with regard to the tensions of a material. As soon as an external force stresses an object, it begins to react starting from a deformation up to its breakup. Nowadays the term "stress" is often improperly referred to situations and events that cause discomfort or disturbance. However, we cannot avoid stress because it is the body's physiological response to every request of modification made on it. This behavior, also referred as "General Adaptation Syndrome", is a non-specific reaction to any request (stressor) from the external or internal environment, involving all the biological systems of the body. The individual may well adapt to a stressor for the short term (eustress); but prolonged and/or overly intense exposure to stressor can produce negative reactions of adjustment (distress). The subjective attribution of value to events, justifies the distress coming from individual sensation of not being able to respond to requests or not to be up to expectations [5].

c) sleep and dream: starting with the premise that we, on average, spend one third of our lives sleeping, the importance of sleep continues to be underestimated. Since the beginning of history, and likely even earlier, sleep and dreams have always interrogated the intelligence by taking on a sacred, spiritual, and ultimately therapeutic value. In modern times, we are constantly learning more about both the micro and macrostructures of sleep by the analysis of the EEG and correlating them to all the major functional organic parameters (Polysomnography). About $27 \%$ of the general population manifests sleep disorders for physical illness or invasive therapies [6].

d) oneiric activity and stress at work: the abuse of the term stress can easily lead up us to fail to distinguish it from simple fatigue. However, while fatigue can be resolved with a quality rest or drugs, on the other hand the real distress, which is derived from an unconscious and pathogenic conflict, can only be resolved with a psychodynamic psychotherapy. Needless to physically tire ourselves in the hope of finally being able to rest. Insomnia, which is divided into the initial, intermediate and terminal types, is a phenomenon often due to certain mental disorders, like anxiety and depression; but sometimes, in proportion to the extent of our fatigue, we repeat night-time awakenings 
in order to fall back asleep and so reproduce the initial deeper and more restful stages of sleep (III-IV). While it is well known that we all dream every night during the REM stages of sleep, we sometimes reawake only with an either pleasant or unpleasant feeling left behind by the dream itself, without remembering any of its contents. More typically, however, fragments or complete stories of ordinary or extravagant contents remain in our minds. Whatever the case, it has been clinically proven that we only remember the parts of the dream that need to be brought from the unconscious to conscious level. This allows the true meanings of these symbolic elements to be processed and to be finally included in the free and complete expression of our Personal Identity. The contents of dreams are always used by the individual exclusively for their value in terms of symbolic personal or universal meaning, and never represent themselves. The references, therefore, are never explicitly in play, and the subjects employed as symbols must be unequivocally excluded from any hypothetical responsibility for the unconscious conflict in progress. This is particularly true also when we, as creators of the dream, represent ourselves as protagonists of situations that in reality we would avoid. Furthermore, in our conscious experience there is an inevitable contrast between the need for safety and security and the limits imposed by space, time and emotional ties. These constraints do not exist in the unconscious, and we can achieve our goals freely, by discovering that there is another way to interpret reality in which the experience regains its original symbolic meaning and ceases to be neurotic or dissociative. One of the significant symbols that is universally used to deal with the actual stress acquired from our relationship with safety and security is that of the thieves. These "professionals" of hazardous unpredictability are often adopted as the feared protagonists of our dreams. In this sense, the goal of the dreams' creator (the real Ego) is to disavow the declared uncertainty of the actor (the presumed Ego), which is often derived from an anxious expectation induced from the outside, as is thus seen as a cause for distress. In the particular case of security guards, who are required to expose themselves to risk in order to protect others, the universal symbol of the criminal overlaps with that of the goal of their own work, or rather the referent for guaranteeing survival. This coincidence, which represents a typical case of symbolic fusion, risks of confusing the sense of our personal security with that of protection required by the customer. The oneiric representation of such characters, therefore, is aimed at distinguishing between the fear for one's own survival and the empathy felt for the customer, as well as to ease the pressure of this stressful and ambiguous distinction. In short, when faced with a dream in which the presumed Ego fears the presence of thieves, kidnappers, or criminals, this situation can be described as one of stress due to vicarious traumatization. In fact, the real Ego of the dreamer not only doesn't fear these symbols as personal referents of unpredictability, but 
since they also represent the fear of his customers, or rather of those who expect something of professionally valid from him, his positive self-esteem is also increased by this professional expectations. Finally, in addition to confirm their irreplaceable adaptogenic role, the psychodynamic processing of these oneiric representations in clinical practice employs them as credible evidence for the existence of the unknown part of ourselves represented by the real Ego, which serves as the immutable and irreplaceable guide to both the discovery and the full expression of our Personal Identity [7]. Based on the above four references, the aim of our investigation-search was to gather preliminary information on the existence of correlations between sleep variables, including any particular dream, and the risk of stress related work accidents recognizable in sentinel events as in factors of content and context.

\section{Method}

The research carried out by our team was divided into five stages.

Step 1: to find a company interested in participating in the study.

The proposal to cooperate with our study was accepted by Sicuritalia S.p.A., an Italian organization, founded in 1956, leader in the field of security services and facility management. Sicuritalia is the only Italian company that handles the security services through a coordinated and unified management. The four main areas of its private, fixed or dynamic security services, all equipped with the most advanced technology, are: private security systems; surveillance; investigation; private intelligence and security informatics. In addition, Sicuritalia carries out activities of Facility Management, for storage, handling and the best use of corporate assets and occupied spaces. To directly organize and coordinate its customers Sicuritalia uses a software call "GSS Web" that, monitoring in real-time the activities contracted, allows the creation of a common data base and great homogeneity of the procedures.

Step 2: collection of the documentation for a comparative analysis.

For this purpose were carried out the following two activities:

a) selection of samples between 6 homogeneous macro tasks present in the risk assessment document of Sicuritalia: clerk; operational center attendant; monitoring and first responder service; anti-theft service; sampling values service; head of security service.

b) application and analysis of data related to the selected samples organized for homogeneous groups. In particular, the assessment of work related stress data, referring to the three-year period 2011-2013, associated with anonymous personal data.

Step 3: preparation of questionnaires to administer to the sample.

To better investigate the sample, already characterized by a medium-level of stress, it was proposed the administration of Pittsburg Sleep Quality Index 
(PSQI) (modified in 15 item) test.

Step 4: administration of questionnaires to staff.

Step 5: study and analysis of the collected data with the Statistical Package for Social Science (SPSS).

\section{Materials}

a) The questionnaire administered to the two selected samples (operational center attendants and monitoring-first responder staff) was divided into two parts. Part 1: anonymous personal specifications of individual employees' age; school attendance; sex; nationality (if non-Italian specifying how many years in Italy); position in job seniority; length of service in the company. Part 2: modified PSQI. About the latter, the scales for the evaluation of sleep disorders, by exploring the symptoms and not the etiology, are commonly addressed to point out primary and secondary dissonnie only. The most recent scales, validated versus polysomnographic data, are considered rapid and non-invasive screening tools to identify patients with sleep disorders to submit, if necessary, to more complex and in-depth investigations. In particular, the Pittsburgh Sleep Quality Index - PSQI [8] is a self-assessment scale that discriminates between "good" and "bad" sleepers and provides an easy-to-use list of self-explanatory elements for the clinician and researcher. It provides also a rapid assessment, clinically useful, of the different conditions that may impair the quality of sleep, because it derives its item from clinical experience of sleep disorders. The test takes into consideration the last month and so permits to distinguish transient from persisting sleep problems. In order to properly carry out our research work, the original PSQI test (15 item, 5 of which, reported, for clinical information only, by the eventual bed partner, do not enter in the total score) has been modified in 15 item all evaluated by the compiler. In fact, the original PSQI basement was complemented by 4 item dedicated to investigate the possible dream representation of works elements (number 12-13-14) or thieves (number 15) as an expression of symbolic referents. The overall score of the PSQI is grouped into 7 composite item, rated on a scale from 0 to 3 , whose sum can go from 0 to 21, with a score higher than 5 considered indicative of sleep disorders. In particular, these composite 7 item represent: subjective sleep quality, sleep latency, sleep duration, habitual sleep efficiency, sleep disorders, the use of hypnotic drugs and noise during the day. In this respect, the authors are particularly satisfied to have standardized a valid and reliable psychometric tool for monitoring the sleep quality.

b) Data collected from the company's injury and disease register.

c) Demographic indicators of working forces engaged for qualification in the farm, including assessments of turn-over and full results of work stress risk assessments - related (breakdown of accident indices, context and content factors), both organized as the previous data for homogeneous groups. 


\section{Preliminary analysis of normalcy}

To perform preliminary analysis of normalcy on our samples were separately used frequency functions of Statistical Package for Social Science (SPSS) [9]. In particular, we carried out a:

a) reliability analysis of PSQI (Cronbach's $\alpha$ calculation). From the results obtained by using SPSS we found a Cronbach's $\alpha$ of 0.851 for central operational personnel, and of 0.86 for the zone operators (https://statistics.laerd.com/), confirming a good test trust. In fact as good reliability index of the test, individual item responses for the two samples had not a high variance;

b) preliminary factorial analysis. From the SPSS factorial analysis, we identified the following 3 significant factors: sleep quality, sleep latency and duration of sleep, with an overall explained variance above $70 \%$, that confirms the good internal consistency of the instrument;

c) preliminary analysis of normality in order to use the two-tailed tests of Pearson. The results showed only in the case of need of medicines to sleep, asymmetry and kurtosis that deviate from normal over the recommended range [10] (Table 1 and Fig. 1);

d) Pearson's correlation analysis. The fact that, with the exception of need meds to sleep, all other distributions approach the normal distribution, allows us to use Pearson's index to explore the possible correlation between the areas of: sleep quality; sleep latency; duration of sleep; sleep efficiency; sleep disturbance; day dysfunction due to sleepiness; sleep disturbance; dream activity due to work (Table 2). In addition, we can also correlate demographic variables (education and age with days of injury and disease 2010-13); in fact age and school attendance curves follow a normal trend (Figs 2 and 3 ).

Table 1: Analysis of normality: asymmetry and kurtosis.

\begin{tabular}{|l|c|c|}
\hline & KURTOSIS & ASYMMETRY \\
\hline Sleep quality & 0.17 & 0.46 \\
\hline Sleep latency & 0.54 & -0.83 \\
\hline Duration of sleep & 0.59 & -0.84 \\
\hline Sleep efficiency & 0.69 & 0.35 \\
\hline Sleep disturbance & 0.03 & 0.65 \\
\hline Need meds to sleep & 4.06 & 17.13 \\
\hline Day disfunction due to sleepiness & 0.35 & 0.69 \\
\hline Sleep disturbance & 0.35 & 0.34 \\
\hline Dream activity due to work & -0.38 & 0.65 \\
\hline
\end{tabular}




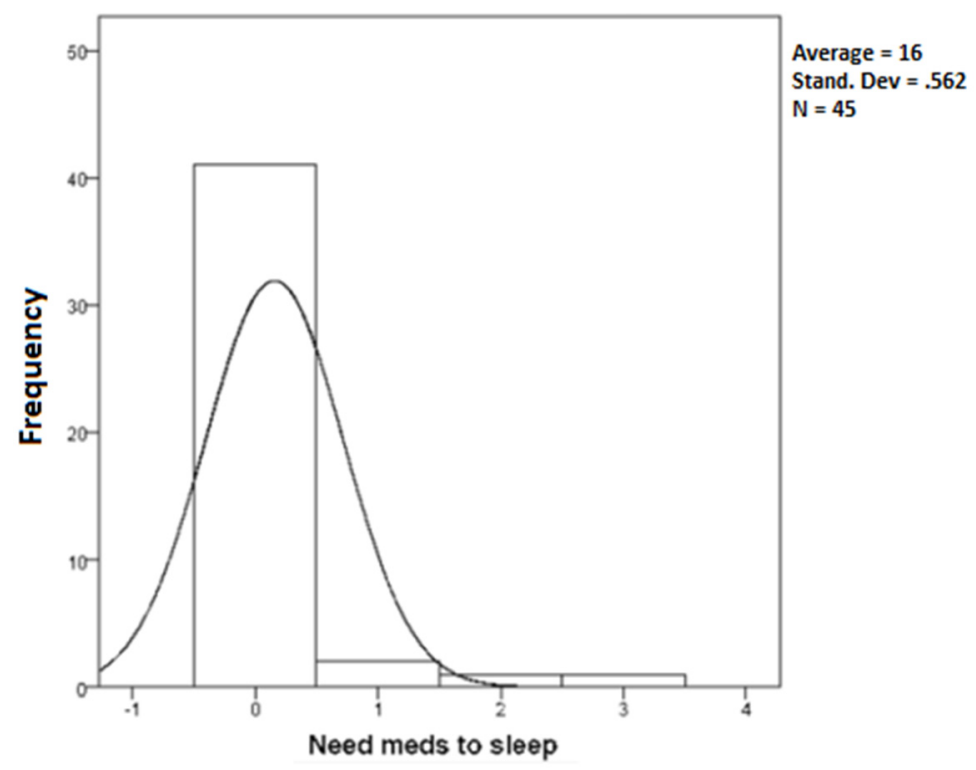

Figure 1: Need of meds to sleep deviation from normal.

Table 2: $\quad$ Pearson's correlation values.

\begin{tabular}{|l|c|}
\hline Sleep quality & 0.6 \\
\hline Sleep efficiency & 0.48 \\
\hline Sleep disturbance & 0.41 \\
\hline Dream activity due to work & 0.4 \\
\hline
\end{tabular}

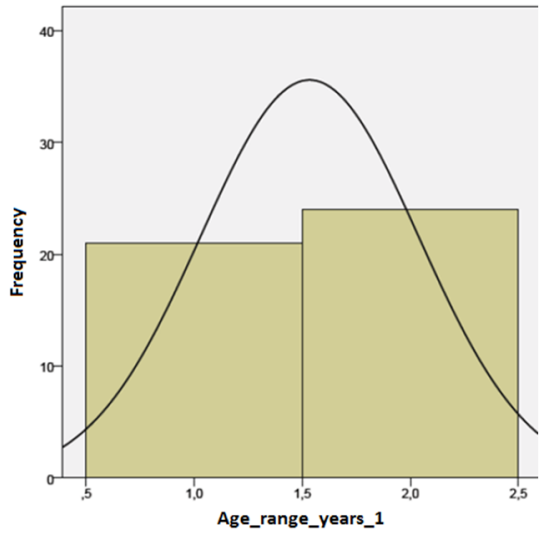

Figure 2: Trend of age curve.

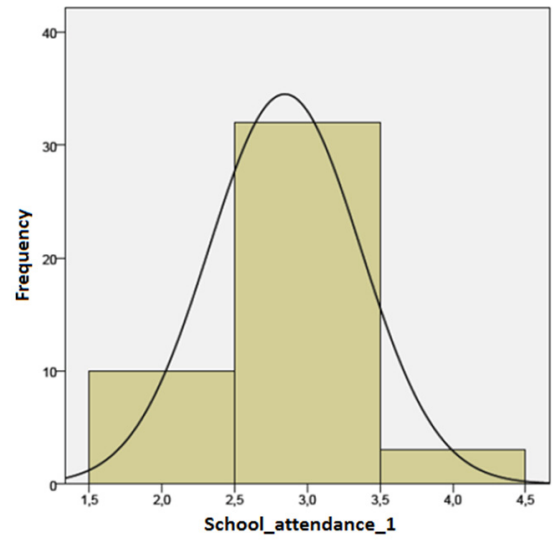

Figure 3: Trend of school attendance. 
e) T-test and ANOVA univariate analysis. In order to have a double check of the results, it was performed a comparison of SPSS averages functions (http://www.cs.uu.nl//SPSS/spss5.pdf) with the t-Test and the ANOVA univariate analysis, because our models involve a single dependent variable. The analyses of personal data and that of results of PSQI scales were carried out together for the two samples, and from the results, we deduce that they both have not a statistically different behavior.

\section{Results and discussion}

We recall that the questionnaire administered to the analyzed sample was divided into the following two parts: Part 1: personal data (anonymous); Part 2: modified in 15 items PSQI. We also had general register of data associated to days for injuries and for diseases in the last three years. The samples analyzed, consisted of less than 100 subjects (45 for each group) respectively attendants to operational center and to monitoring and first responders service; the particular criticality of both these tasks lies in the activities carried out as security guards and with high-risk. In addition, the shifts, including night shift, further influence the quality of sleep. With regard to the issues related to fall asleep, generally wake time exceeds 30 minutes in more than $50 \%$ of the older subjects.

In our research, the matter of injury has been standardized through specific indicators related to number of occurring occasions. Settlement data, stratified for homogeneous groups consistent with definitions used in sampling risk protocols of work-related stress, were obtained from the company's accident register, the National Institute for Insurance Against Industrial Injuries (INAIL) databank and/or periodic statistics publications related to specific production sectors. The indicators considered in our analysis are:

a) the specific injury rate for homogeneous groups of workers, as obtainable from the company's accident record, stratified by level of consequential (mild, serious or fatal outcome);

b) the industry's injury rate, as determined by the INAIL $\mathrm{db}$ or other sources of free access;

c) the average level of damage associated with each category of damage, normalizing the injury in lost work days.

In the analysis, performed with the t-Test and the ANOVA in order to have a double check of results, we basically identified 3 significant factors from the PSQI administration: sleep quality, sleep latency and duration of sleep. This result, with an overall variance explained above $70 \%$, confirms a good internal consistency of the utilized instrument and also demonstrates that the two samples have not a statistically different behavior; in fact for both it is manifest a Pearson correlation of 0.5 .

Actually, to the reduction of the Duration of Sleep can contribute even physical problems, drugs and metabolism. In addition, another situation that can aggravate insomnia in adults is the tendency to doze off during the day that increases with age. This trend can be fostered by lack of interest for external 
stimuli (ipovisus or hearing loss) or physical limitation to movement with confinement of the elderly at home. In particular the latency delay before falling asleep (sleep latency or initial insomnia) represents the most frequently complained about noise from older. Our data also highlight direct correlation between sleep duration and the amount of time to fall asleep. The higher the sleep duration, the greater the time to fall asleep. An important comment deserves the correlation between age and working days lost for injury: the significance is next to the value of verification (0.05). Then the result of Pearson's correlation (0.28) should not be despised. The phenomenon that emerges is that, with increasing age, there is a greater propensity to days lost for injury. The result is consistent with the literature in the field and consistent with the INAIL data 2007-2011. Even though in our samples there are only 4 people over the age of 50 nevertheless they are on average with the INAIL data of correlation, in contrast to what happens for the correlation between old age and illness. Moreover, as there is no correlation between the longest serving (job seniority) and days lost for injury/illness we can assume that the accident data analyzed are the result of objective person's age and wear not work. Another interesting correlation is evident among those who do work-related dreams, all of whom claim to be more subject to problems during the day derived from drowsiness and who must resort to the use of medications for sleeping.

\section{Conclusion}

The significant association found in our research between the occurrence of the work environment in dreams and the tendency to increasing insomnia (sleep latency), directly correlated with aging, as the accidents at work, but not with the longest company serving or illness, suggests an exciting new symbolic meaning in work environment dream-like representation. In fact, recalling the assumption that the symbol never refers to itself, but redirects to an implicit reference or, if generally shared, to a universal one, we can say that the dream representing our own working environment allows us to unconsciously face the uncertainty of risks associated with aging. Therefore, the universality of this existential experience allows, through the use of a shared symbolism, the Jungian solution of the distressing possible conflict: avoid not to grow old! In conclusion, from the psychodynamic perspective, our study would find a new universal symbol for the analytical processing of dreams at the service of the real Ego for the discovery and the full expression of one's Personal Identity.

\section{References}

[1] Cox T. Griffiths A.J. "The assessment of psychosocial hazard at work" Cooper CL (editors) 1995.

[2] Cox T., Griffiths A.J., Barlow C., Randall R., Thomson T \& Rial Gonzales E. "Organisational intervention for work stress: a risk management approach” Sudbury HSE Books 2000. 
[3] Segura O. "Burnout: concepts and implications affecting public health" Biomedica. 34(4):535-45. Dec 2014.

[4] Tomei G.1., Cinti M.E., Sancini A., Cerratti D., Pimpinella B., Ciarrocca M, Tomei F, Fioravanti M. "Evidence based medicine and mobbing." G Ital Med Lav Ergon. 29(2):149-57, Apr-Jun 2007.

[5] Selye H. "Stress and the general adaptation syndrome" BMJ 4667:13831392. 1950.

[6] Winkler A., Rief W. "Effect of Placebo Conditions on Polysomnographic Parameters in Primary Insomnia: A Meta-Analysis". Sleep. 00487-14. Nov $1,2014$.

[7] Borghini F., Garzia F., Borghini A., Borghini G. "Psychology of Safety \& Security, Emergency and Risk", WIT Press (in print).

[8] Buysse D.J., Reynolds C.F., Monk T.H., Berman S.R., Kupfer D.J. “The Pittsburgh Sleep Quality Index (PSQI): A new instrument for psychiatric research and practice" Psychiatry Research, 28(2), 193-213, 1989.

[9] Nie N.H. "SPSS Statistical Package for the Social Sciences" Mc Graw Hill NY, 1975.

[10] Hae-Young Kim "Statistical notes for clinical researchers: assessing normal distribution using skewness and kurtosis". Restor Dent Endod.; 38(1): 52-54, Feb 2013. 